\title{
On the Deficiency of the Ott-Clemmow Modified Saddle-Point Method in the Sommerfeld Half-Space Problem
}

This paper was downloaded from TechRxiv (https://www.techrxiv.org).

\section{LICENSE}

CC BY 4.0

SUBMISSION DATE / POSTED DATE

24-10-2021 / 29-10-2021

\section{CITATION}

Michalski, Krzysztof; Mosig, Juan (2021): On the Deficiency of the Ott-Clemmow Modified Saddle-Point Method in the Sommerfeld Half-Space Problem. TechRxiv. Preprint.

https://doi.org/10.36227/techrxiv.16863667.v1

$\mathrm{DOI}$

10.36227/techrxiv.16863667.v1 


\title{
On the Deficiency of the Ott-Clemmow Modified Saddle-Point Method in the Sommerfeld Half-Space Problem
}

\author{
Krzysztof A. Michalski, Life Fellow, IEEE, and Juan R. Mosig, Life Fellow, IEEE
}

\begin{abstract}
We investigate the accuracy of the multiplicative and additive modified saddle-point integration methods, as applied to the Sommerfeld problem of a vertical Hertzian dipole radiating over a lossy half-space. It is demonstrated that the first-order additive method leads to the same asymptotic field representation irrespective of whether a positive or a negative dipole image is employed, whereas the multiplicative variant yields different results depending on which image is utilized. With the positive image, the well-known Norton formula is obtained, but the firstorder multiplicative method fails when the negative image is used.
\end{abstract}

Index Terms-Sommerfeld half-space problem, Hertzian dipole, modified saddle-point integration method, Norton surface wave, near-ground propagation

\section{INTRODUCTION}

$\mathbf{T}$ HE Sommerfeld half-space problem has a long history fraught with some controversies [1, Sec. 4.10], [2], [3], caused by an elusive error in the asymptotic field expansion developed by Sommerfeld in his seminal contribution [4], even though he presented a correct formula in a follow-up paper [5]. Sommerfeld and his early followers relied in their derivations on ingenious but mostly ad hoc mathematical devices, usually based on the high-contrast assumption appropriate for lossy earth at low frequencies. These methods were also restricted to far zone field points near the interface, until Norton [6] devised a formulation valid for all elevation angles, by extending the methods of van der Pol [7] and Wise [8]. The first systematic asymptotic procedure was presented by Ott [9], who adopted a multiplicative modified saddle-point procedure introduced in a different context by Pauli [10]. This modification was required due to the close proximity of the pole of the reflection coefficient - often referred to as the Sommerfeld pole- to the saddle point, which occurs for low elevation angles in the high-contrast case. A similar pole treatment, equivalent to the first-order Pauli-Ott method, was subsequently developed by Clemmow [11], [12], and was later employed by Wait [13], [14]. In the more recent literature [3], [15], the multiplicative method has been largely superseded by an additive pole

Manuscript received September 3, 2019; accepter December 14, 2019. Date of publication January 15, 2020; date of current version January 15, 2020.

Krzysztof A. Michalski is with Texas A\&M University, College Station, TX 77843-3128, USA (e-mail: k-michalski@tamu.edu).

Juan R. Mosig is with EPFL, Lausanne, CH-1015, Switzerland (e-mail: juan.mosig@epfl.ch).

Color versions of one or more of the figures in the paper are available online at http://ieeexplote.ieee.org.

Digital Object Identifier xx.xxxx/TAP.2019.xxxxxxx treatment introduced by van der Waerden [16] and elaborated by Felsen and Marcuvitz [17, Ch. 4]. Michalski and Lin [18] have recently demonstrated that these methods are equivalent, provided that an infinite number of terms are included in the expansions. Since in practice only the leading term is usually retained, the resulting approximations are different-as was also pointed out by van der Waerden [16].

When the asymptotic methods are applied to the Sommerfeld half-space problem, the direct and the positive or negative image dipole fields are usually first extracted in a closed form. The purpose of this paper is to investigate and compare the performance of the first-order additive and multiplicative variants of the saddle-point method with the positive and negative image formulations. It is found that, as might be expected, the additive method yields the same asymptotic field, irrespective of which image, positive or negative, is extracted. Surprisingly, however, the multiplicative variant leads to different outcomes in these two cases, which to our knowledge has not been documented before. In fact, whereas the well-known Norton formula is obtained with the positive image, the result with the negative image is inaccurate and this formulation completely fails in the on-surface transmitter-receiver configuration.

\section{Formulation}

We consider the Sommerfeld half-space problem illustrated in Fig. 1, where a vertical Hertzian dipole (the $e^{j \omega t}$ time convention is assumed) with a current moment $I \ell$ radiates in air above a lossy ground. The media contrast will be denoted by $\epsilon=\varepsilon_{2} / \varepsilon_{1}$ and the meaning of other parameters is selfexplanatory. For this problem, the $z$ component of the electric

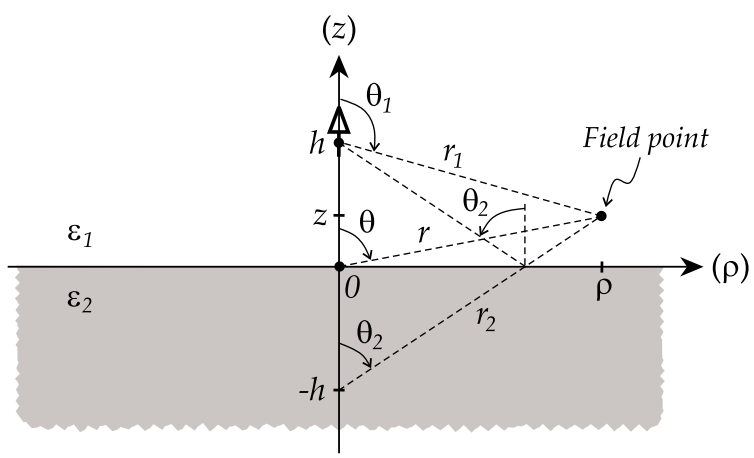

Fig. 1. Schematic of Hertzian dipole radiating over a lossy half space. Indicated is also the image dipole location. 
field in the upper half-space may be expressed as

$$
E_{z 1}=-j k_{1}^{2} \eta_{1} \frac{I \ell}{4 \pi} \mathcal{E}
$$

where $k_{1}$ and $\eta_{1}$ are the wavenumber and intrinsic impedance of the upper medium (air in our case), and $\mathcal{E}$ is the normalized field given as

$$
\mathcal{E}=\mathcal{E}^{(1)} \pm \mathcal{E}^{(2)} \mp \mathcal{E}_{\mp}^{C}
$$

where

$$
\mathcal{E}^{(i)}=\left[\sin ^{2} \theta_{i}-\left(1-3 \cos ^{2} \theta_{i}\right)\left(\frac{j}{\Omega_{i}}+\frac{1}{\Omega_{i}^{2}}\right)\right] \frac{e^{-j \Omega_{i}}}{\Omega_{i}}
$$

with $\Omega_{i}=k_{1} r_{i}, i=1$ or 2 , are the direct and image dipole fields in an infinite homogeneous space, respectively, and $\mathcal{E}_{\mp}^{C}$ is the corresponding "correction field" accounting for the effect of the lower half-space. The latter may be expressed in the angular spectrum plane as [19]

$$
\mathcal{E}_{\mp}^{C} \approx \frac{1}{\sqrt{2 \pi j \Omega_{2}}} \int_{C_{\mathrm{SDP}}} g_{\mp}(\xi) e^{-j \Omega_{2} \cos \left(\xi-\theta_{2}\right)} d \xi
$$

where $C_{\mathrm{SDP}}$ is the steepest descent path (SDP) in the complex $\xi$ plane with the saddle point at $\xi=\theta_{2}$, and where

$$
g_{\mp}(\xi)=f(\xi)[1 \mp \Gamma(\xi)]
$$

with

$$
f(\xi)=\sin ^{2} \xi \sqrt{\frac{\sin \xi}{\sin \theta_{2}}}
$$

and $\Gamma(\xi)$ is the reflection coefficient for parallel-polarized waves, defined as

$$
\Gamma(\xi)=\frac{\cos \xi-\Delta(\xi)}{\cos \xi+\Delta(\xi)}
$$

where

$$
\Delta(\xi)=\frac{\kappa(\xi)}{\epsilon}, \quad \kappa(\xi)=\sqrt{\epsilon-\sin ^{2} \xi} .
$$

The denominator of the reflection coefficient has a root at $\xi_{p}$, which satisfies the relations

$$
\sin \xi_{p}=\sqrt{\frac{\epsilon}{\epsilon+1}}, \quad \cos \xi_{p}=-\frac{1}{\sqrt{\epsilon+1}}
$$

and contributes a pole to the integrand of (4), which may influence the field behavior. Note that (4) is approximate as a result of the replacement of the Hankel function $H_{0}^{(2)}\left(\Omega_{2} \sin \theta_{2} \sin \xi\right)$ by its large-argument form.

The upper and lower signs in (4)-(5) correspond to the positive and negative image dipoles, respectively, and should be used consistently in all equations. Although the extraction of a closed-form image term is not absolutely necessary and not always helpful [20], it is a common practice in the literature of the Sommerfeld half-space problem. Both positive and negative image conventions have been employed and, in theory, the outcome of (2) should be the same irrespective of whether the upper or lower signs are selected, and thus this choice would seem to be just a matter of personal taste. In practice, however, these results may be different, depending on the asymptotic procedure employed —as will be demonstrated in due course.
To facilitate the asymptotic evaluation of the integral in (4), we perform a mapping into the $s$ plane by the transformation

$$
\cos \left(\xi-\theta_{2}\right)=1-j s^{2}
$$

with the Jacobian

$$
\frac{d \xi}{d s}=\frac{\sqrt{2 j}}{\cos \frac{\xi-\theta_{2}}{2}}=\frac{\sqrt{2 j}}{\sqrt{1-j \frac{s^{2}}{2}}}
$$

and we obtain

$$
\mathcal{E}_{\mp}^{C} \approx \frac{e^{-j \Omega_{2}}}{\sqrt{\pi \Omega_{2}}} \int_{-\infty}^{\infty} G_{\mp}(s) e^{-\Omega_{2} s^{2}} d s
$$

where

$$
G_{\mp}(s)=\left.\frac{1}{\sqrt{2 j}} \frac{d \xi}{d s} g_{\mp}(\xi)\right|_{\xi_{\mathrm{SDP}}}
$$

in which $\xi_{\mathrm{SDP}}$ defines $C_{\mathrm{SDP}}$ in terms of $s$ [21]. The transformation (10) thus maps $C_{\mathrm{SDP}}$ into the real axis of the $s$ plane and the saddle point $\theta_{2}$ into its origin $s=0$. Furthermore, the pole $\xi_{p}$ is mapped into $s_{p}$ via

$$
s_{p}^{2}=-j\left[1-\cos \left(\xi_{p}-\theta_{2}\right)\right] .
$$

It should be noted that $s_{p} \rightarrow 0$ when $\theta_{2} \rightarrow \xi_{p}$, where $\xi_{p}$ is very close to $\pi / 2$ in the high-contrast case, in view of (9). Therefore, the pole can become very close to the saddle point for elevation angles near the interface, and this poses a difficulty, which can be remedied by the modified saddle-point integration method, either additive or multiplicative.

\section{A. Additive pole treatment}

In the additive variant we subtract and add the pole term as

$$
\begin{aligned}
G_{\mp}(s) & =\left[G_{\mp}(s)-\frac{r_{p}}{s-s_{p}}\right]+\frac{r_{p}}{s-s_{p}} \\
& \approx\left[g_{\mp}\left(\theta_{2}\right)+\frac{r_{p}}{s_{p}}\right]+\frac{r_{p}}{s-s_{p}}
\end{aligned}
$$

where in the approximate expression we have evaluated the first term at the saddle point $s=0$, which corresponds to $\xi=\theta_{2}$. The residue $r_{p}$ is readily found as

$$
r_{p}=\mp f\left(\xi_{p}\right) R_{p}
$$

where $R_{p}$ is the residue of $\Gamma(\xi)$ at $\xi_{p}$ and is given as

$$
R_{p}=\frac{2 \epsilon \sqrt{\epsilon}}{\epsilon^{2}-1} .
$$

We next substitute the approximation (15) into (12) and use the integral results of Appendix A to obtain

$$
\begin{aligned}
\mathcal{E}_{\mp}^{C}(s) \approx & \sin ^{2} \theta_{2}\left[1 \mp \Gamma\left(\theta_{2}\right)\right] \frac{e^{-j \Omega_{2}}}{\Omega_{2}} \\
& \mp \sin ^{2} \theta_{2} \Upsilon\left(\theta_{2}\right) \mathcal{F}(p) \frac{e^{-j \Omega_{2}}}{\Omega_{2}}
\end{aligned}
$$

where

$$
\Upsilon\left(\theta_{2}\right)=\frac{R_{p}}{\sqrt{2 j} s_{p}}\left(\frac{\sin \xi_{p}}{\sin \theta_{2}}\right)^{5 / 2}
$$

and

$$
\mathcal{F}(p)=1-j \sqrt{\pi p} w(-\sqrt{p})
$$


is the Sommerfeld-Norton attenuation function, where $p$ is the numerical distance defined in (41). Note that in view of (14) and (9) we may express $p$ as

$$
p=-j \Omega_{2}\left(1-\sqrt{\frac{\epsilon}{\epsilon+1}} \sin \theta_{2}+\frac{\cos \theta_{2}}{\sqrt{\epsilon+1}}\right) .
$$

If we now substitute (18) into (2) and neglect the second- and third-order terms in (3), we obtain

$$
\mathcal{E} \approx \mathcal{E}_{G O}+\sin ^{2} \theta_{2} \Upsilon\left(\theta_{2}\right) \mathcal{F}(p) \frac{e^{-j \Omega_{2}}}{\Omega_{2}}
$$

where $\mathcal{E}_{G O}$ is the first-order geometrical-optics field, given as

$$
\mathcal{E}_{G O}=\sin ^{2} \theta_{1} \frac{e^{-j \Omega_{1}}}{\Omega_{1}}+\sin ^{2} \theta_{2} \Gamma\left(\theta_{2}\right) \frac{e^{-j \Omega_{2}}}{\Omega_{2}} .
$$

Note that in this additive variant the same asymptotic field expression (22) arises irrespective of whether the upper or lower signs are employed in (2). The geometrical-optics field (23) vanishes when both the transmitter and the receiver are on the interface, since in this case $\theta_{1}=\theta_{2}=\pi / 2, r_{1}=r_{2}=\rho$, and $\Gamma\left(\theta_{2}\right)=-1$. For this reason, the first term in (22) is also referred to as the space wave, and the second term as the surface wave [22].

\section{B. Multiplicative pole treatment}

In the multiplicative variant, we multiply and divide $G_{\mp}(s)$ by $\left(s-s_{p}\right)$ as

$$
G_{\mp}(s)=\left[\left(s-s_{p}\right) G_{\mp}(s)\right] \frac{1}{s-s_{p}} \approx\left[-s_{p} g_{\mp}\left(\theta_{2}\right)\right] \frac{1}{s-s_{p}}
$$

where again we have approximated the first term by its value at the saddle point $s=0$. Upon substituting into (12) and referring to Appendix A, we now obtain

$$
\mathcal{E}_{\mp}^{C}(s) \approx \sin ^{2} \theta_{2}\left[1 \mp \Gamma\left(\theta_{2}\right)\right][1-\mathcal{F}(p)] \frac{e^{-j \Omega_{2}}}{\Omega_{2}}
$$

which should be compared with (18) in the additive variant. If we now use this approximation in (2), we arrive at

$$
\mathcal{E} \approx \mathcal{E}_{G O} \pm \sin ^{2} \theta_{2}\left[1 \mp \Gamma\left(\theta_{2}\right)\right] \mathcal{F}(p) \frac{e^{-j \Omega_{2}}}{\Omega_{2}}
$$

which clearly yields a different asymptotic field, depending on whether the upper or lower signs are selected. In the former case (positive image) we obtain

$$
\mathcal{E} \approx \mathcal{E}_{G O}+\sin ^{2} \theta_{2}\left[1-\Gamma\left(\theta_{2}\right)\right] \mathcal{F}(p) \frac{e^{-j \Omega_{2}}}{\Omega_{2}}
$$

whereas the latter choice (negative image) results in

$$
\mathcal{E} \approx \mathcal{E}_{G O}-\sin ^{2} \theta_{2}\left[1+\Gamma\left(\theta_{2}\right)\right] \mathcal{F}(p) \frac{e^{-j \Omega_{2}}}{\Omega_{2}} .
$$

It should be noted that the multiplicative pole treatment described here is more direct than those of Ott and Clemmow, who effect the pole cancellation in the $\xi$ domain. Ott used as the canceling multiplier the function $\left[\cos \left(\xi-\theta_{2}\right)-\cos \left(\xi_{p}-\theta_{2}\right)\right]$, while Clemmow employed $\sin \left[\left(\xi-\xi_{p}\right) / 2\right]$. The second- and third-order multiplicative modified saddle point methods are more complex [18] and are summarized in Appendix B.

\section{Analysis of the variants}

Since the three asymptotic field expressions (22), (27) and (28) were derived under the same assumption that $\Omega_{2} \gg 1$, it may be disconcerting that they should yield different results for a large, but finite $\Omega_{2}$. We note that all three formulas comprise the same space wave term $\mathcal{E}_{G O}$, hence the differences come from the surface wave terms, which all include the attenuation function $\mathcal{F}(p)$ as a factor. In fact, the discrepancy between the multiplicative variants (27) and (28) is found as

$$
\Delta \mathcal{E}=2 \sin ^{2} \theta_{2} \mathcal{F}(p) \frac{e^{-j \Omega_{2}}}{\Omega_{2}} .
$$

That the same additive formula (22) applies irrespective of whether the positive or negative image is employed may testify to its superiority over the two multiplicative variants. Furthermore, unlike the multiplicative variants, this expression comprises the residue of the reflection coefficient at the Sommerfeld pole. The positive-image multiplicative variant (27) is recognized as the formula of Norton [23, Sec. 3.7], except that Norton's numerical distance is given as [24]

$$
p_{N}=-j \Omega_{2} \frac{\left[\cos \theta_{2}+\Delta\left(\theta_{2}\right)\right]^{2}}{2 \sin ^{2} \theta_{2}} .
$$

Although this formula is different from (21), both $p_{N}$ and $p$ reduce to

$$
p_{N} \approx p \approx-j \frac{k_{1} \rho}{2 \epsilon}
$$

in the on-surface transmitter-receiver configuration with high media contrast. Furthermore, we can show that under the same conditions, the coefficients preceding $\mathcal{F}(p)$ in (22) and (27) become identical and equal to 2 , and thus for $\theta_{2}=\pi / 2$ and $|\epsilon| \gg 1$ these variants yield the same result. On the other hand, in the negative-image multiplicative variant (28), the surface wave vanishes in the on-surface configuration, since $\Gamma(\pi / 2)=-1$. We may thus reasonably expect (28) to be less accurate than (27) for higher elevation angles as well.

The interface failure of the negative-image variant may have been the reason why Wait [25, Ch. 2, Sec. 11], [26, Sec. 6.6], unlike Norton [6], exclusively employed the positive image formulation. Wait applied the Clemmow method under the high-contrast assumption, in which case the numerical distance takes the form

$$
p \approx \frac{-j \Omega_{2}}{2}\left(\cos \theta_{2}+\frac{1}{\sqrt{\epsilon}}\right)^{2} .
$$

\section{NumericAl RESUlts AND DISCUSSION}

We illustrate the theoretical developments by numerical results, which are validated against "exact" results generated by real-axis evaluation of the Sommerfeld integrals by numerical quadrature with extrapolation [27], [28], and also against commercial code results generated by FEKO [29]. The relative permittivity of a lossy half-space is given as $\varepsilon=\varepsilon_{r}-j \sigma /\left(\omega \varepsilon_{0}\right)$, where $\varepsilon_{0}$ is the free-space permittivity and $\sigma$ is the conductivity, or as $\varepsilon=\varepsilon_{r}(1-j \tan \delta)$, where $\tan \delta$ denotes the loss tangent. 


\section{A. On-surface transmitter-receiver configuration}

We begin with the on-surface transmitter-receiver configuration and first consider a case where the dipole of Fig. 1 is at the surface of seawater $\left(\varepsilon_{r}=80, \sigma=4 \mathrm{~S} / \mathrm{m}\right)$ with the operating frequency $f=10 \mathrm{MHz}$. For this case, in Fig. 2 we plot the

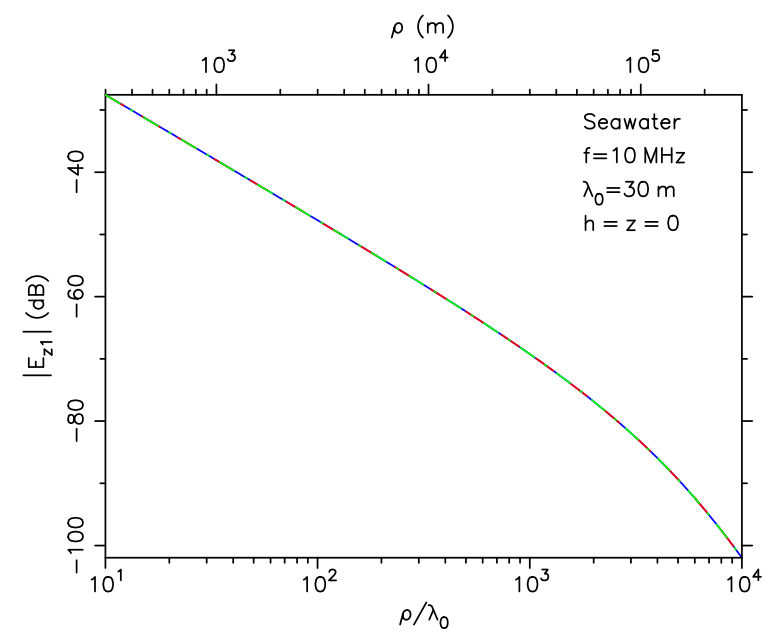

Fig. 2. Plots of $\left|E_{z 1}\right|$ vs. $\rho$ in the on-surface transmitter-receiver configuration for seawater $\left(\varepsilon_{r}=80, \sigma=4 \mathrm{~S} / \mathrm{m}\right)$ at $10 \mathrm{MHz}$. The exact result is indicated by a full blue line, the additive saddle-point method asymptotic field (22) is plotted by a dashed red line, and the positive-image multiplicative method (Norton formulation) field (27) is represented by a dot-dash green line. These plots overlap and are indistinguishable in the figure. The geometrical-optics field (23) is identically zero in the on-surface configuration.

asymptotic field (22), derived by the additive modified saddlepoint method-which we recall is the same irrespective of the whether the positive or negative image is employed-over a three-decade horizontal range $10<\rho / \lambda_{0}<10^{4}$ and note that the field plot initially follows a $-20 \mathrm{~dB} /$ decade asymptote and then transitions to a $-40 \mathrm{~dB} /$ decade asymptote in the far zone. Since the geometrical-optics component $\mathcal{E}_{G O}$ is identically zero in this case, what is plotted in Fig. 2 is exclusively the surface wave part, which we will denote by $\mathcal{E}_{N}$. The two asymptotes are thus determined by the behavior of the attenuation function $\mathcal{F}(p)$, viz., when the numerical distance is small and $|p| \ll 1$, we see from $(42)$ that $\mathcal{F}(p) \approx 1$ and the surface field behaves as $\rho^{-1}$, whereas for $|p| \gg 1$ the leading term in the asymptotic expansion (43) is governed by the $p^{-1}$ term, since the first term is absent in the nonplasmonic case considered here, and the far-zone surface field may be approximated as

$$
\mathcal{E}_{N} \approx-2 j \epsilon \frac{e^{-j k_{1} \rho}}{\left(k_{1} \rho\right)^{2}} .
$$

We may thus take $|p|=1$ as the demarcation point where the transition between the $\rho^{-1}$ and $\rho^{-2}$ asymptotes begins, and from this condition we obtain the "knee value" of $\rho$ as [30]

$$
\rho_{\mathrm{knee}} / \lambda_{0} \approx \frac{|\epsilon|}{\pi}
$$

which in the case of Fig. 2 yields $\approx 2.3 \times 10^{3}$. The average error in the additive formula (22) plotted in this figure is $0.24 \%$. The multiplicative Norton-like formula (27) yields a very similar result, with the error of $0.25 \%$, while the multiplicative negative image variant (28) predicts a zero field and thus fails completely. This is illustrated in Fig. 3, where we plot the relative errors of the first-, second-, and third-order negativeimage multiplicative methods as applied to the case of Fig. 2. Clearly, one has to resort the second-order method if the

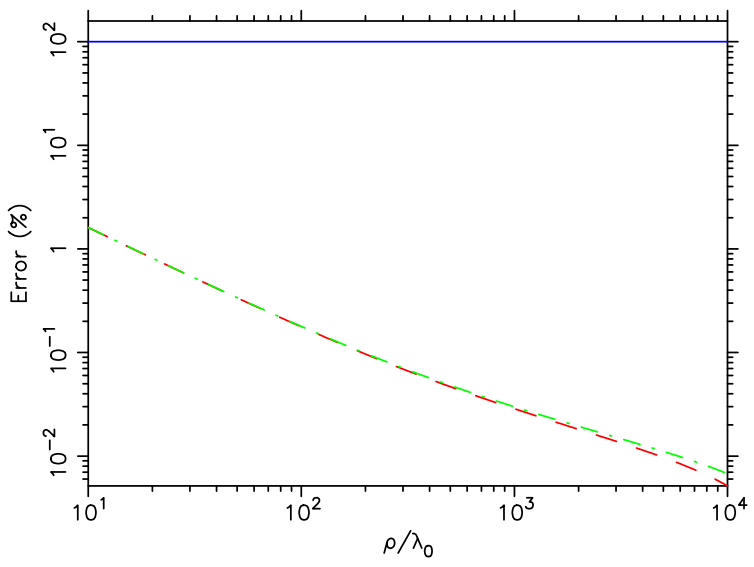

Fig. 3. Plots of the errors of the negative-image multiplicative methods for the on-surface seawater case of Fig. 2. The error of the first-order Ott-Clemmow formula is plotted by a full blue line, whereas the errors of the second- and third-order formulas are plotted by the dashed red and dash-dot green lines, respectively.

multiplicative negative-image dipole is employed. However, the third-order method does not result in further accuracy improvement.

In Fig. 4 we present on-surface configuration results for moist soil $\left(\varepsilon_{r}=15, \tan \delta=10^{-3}\right)$ at $2.4 \mathrm{GHz}$. Note that

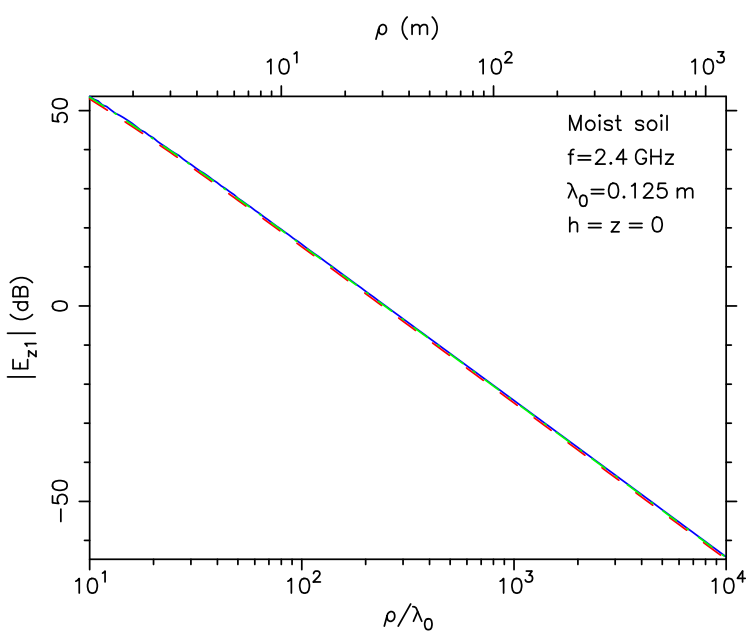

Fig. 4. As in Fig. 2, except for moist soil $\left(\varepsilon_{r}=15, \tan \delta=10^{-3}\right)$ at $2.4 \mathrm{GHz}$.

this is a low-loss, low-contrast medium problem. In this case the knee-point (34) is found at 4.8, hence the field follows the $\rho^{-2}$ asymptote in the entire horizontal range plotted in the figure. The average errors of the additive formula (22) and the multiplicative Norton formula (27) plotted in Fig. 4 are found as $7.2 \%$ and $6.4 \%$, respectively. Hence, the firstorder asymptotic results are considerably less accurate than in the high-contrast case of Fig. 2. The error plots of the multiplicative negative-image methods of the first, second, and third order for the case of Fig. 4 are shown in Fig. 5. Again, 


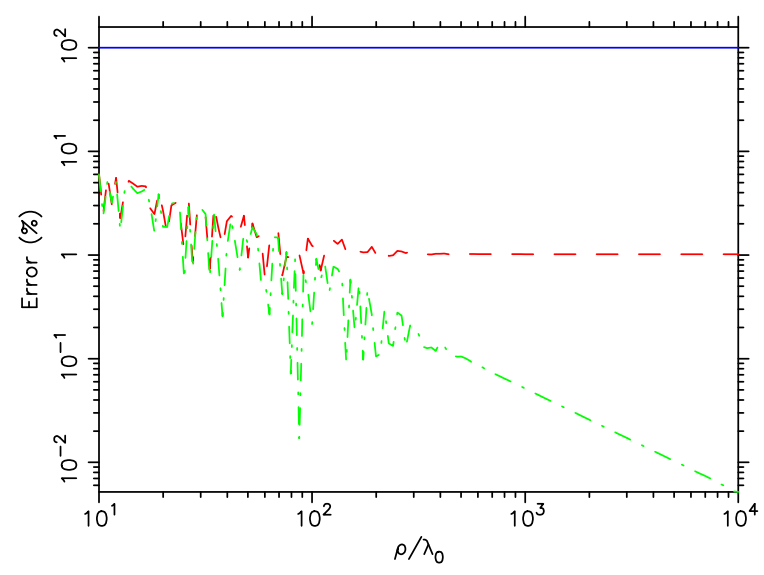

Fig. 5. As in Fig. 3, except for the on-surface moist soil case of Fig. 4.

we note a complete failure of the first-order Ott-Clemmow method, with the error at $100 \%$ over the entire horizontal range, and it appears that one has to resort to the third-order method in this case.

\section{B. Elevated transmitter-receiver configuration}

We next turn attention to the elevated transmitter-receiver configuration, where the geometrical-optics field $\mathcal{E}_{G O}$ is no longer zero. In fact, we can show that [31]

$$
\mathcal{E}_{G O} \approx 2\left[\frac{\epsilon}{\sqrt{\epsilon-1}} k_{1}(z+h)+j k_{1}^{2} z h\right] \frac{e^{-j k_{1} \rho}}{\left(k_{1} \rho\right)^{2}}
$$

when $\rho \gg(z+h)$. It is remarkable that this formula predicts the same $\rho^{-2}$ far-zone field behavior as that in the surface wave (33) and, furthermore, that $\mathcal{E}_{G O}$ grows in magnitude with increasing $z$ and $h$. The relative importance of the surface wave term in the near-ground field of a dipole antenna may thus be evaluated by the ratio [31]

$$
\mathcal{R} \equiv\left|\frac{\mathcal{E}_{N}}{\mathcal{E}_{G O}}\right| \approx \frac{|\epsilon|}{\left|\frac{\epsilon}{\sqrt{\epsilon-1}} k_{1}(z+h)+j k_{1}^{2} z h\right|} .
$$

We may thus take $\mathcal{R} \approx 0.1$ as the cut-off value below which the surface wave contribution is for all practical purposes insignificant with the field behavior solely governed by the geometrical-optics component.

We first return to the seawater case of Fig. 2, but with $h=25 \mathrm{~m}$ and $z=5 \mathrm{~m}$, so that $(h+z) \approx 1 \lambda_{0}$. The corresponding results are plotted in Fig. 6, where we observe that the geometrical-optics field is only significant over the initial part of the horizontal range, hence the field behavior is still dominated by the surface wave term. This should be expected, since $\mathcal{R} \approx 13.6$ in this case. Note that the dipole elevation is moderate in terms of wavelengths, which may explain the similarity between the total field plots in Fig. 6 and Fig. 2. The average errors of the additive variant (22) and the Norton formula (27) are both found to be around $0.25 \%$. The error plots of the first-, second-, and third-order negative-image multiplicative methods for this case are given in Fig. 7. Again, a complete failure of the first-order Ott-Clemmow formula should be noted.

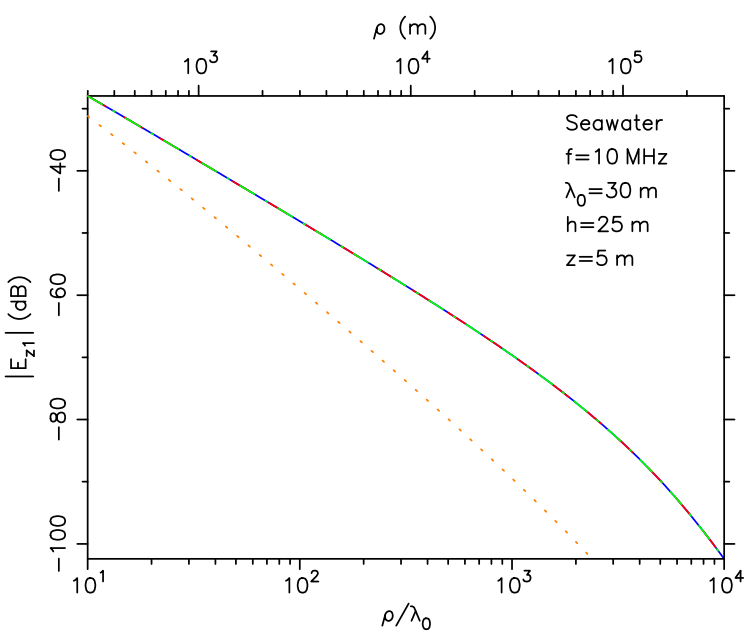

Fig. 6. Plots of $\left|E_{z 1}\right|$ vs. $\rho$ for the seawater case of Fig. 2, but in the elevated transmitter-receiver configuration with $h=25 \mathrm{~m}$ and $z=5 \mathrm{~m}$. The exact result is indicated by a full blue line, the additive saddle-point method asymptotic field (22) is plotted by a dashed red line, and the positive-image multiplicative method (Norton formulation) field (27) is represented by a dot-dash green line. These plots overlap and are indistinguishable in the figure. Included is also a plot of the geometrical-optics field (23), shown by a dotted orange line.

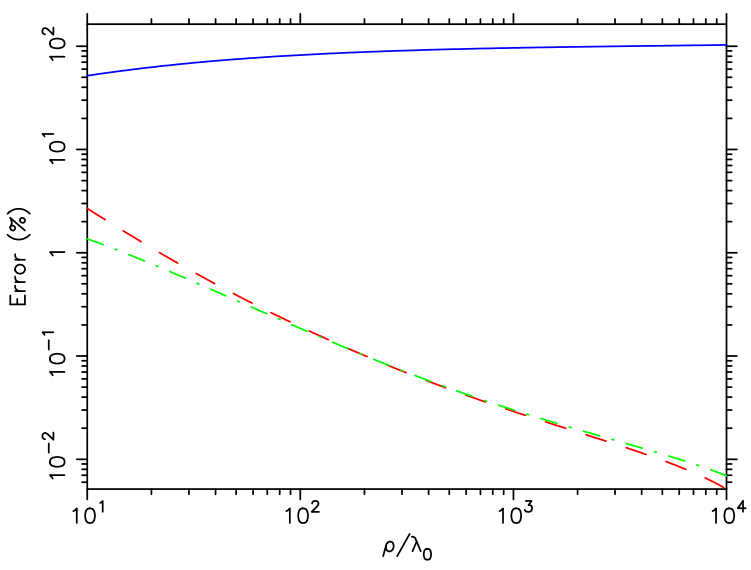

Fig. 7. Plots of the errors of the negative-image multiplicative methods for the elevated seawater case of Fig. 6. The error of the first-order Ott-Clemmow formula is plotted by a full blue line, whereas the errors of the second- and third-order formulas are plotted by the dashed red and dash-dot green lines, respectively.

In the last example we return to the moist soil $\left(\varepsilon_{r}=15\right.$, $\left.\tan \delta=10^{-3}\right)$ at $2.4 \mathrm{GHz}$, but in the elevated transmittedreceiver configuration with $h=2 \mathrm{~m}$ and $z=1 \mathrm{~m}$, so that $(h+z) \approx 24 \lambda_{0}$ [31]. In this case we find $\mathcal{R} \approx 0.003$, hence the surface-wave term should be negligible, with the field behavior dominated by the geometrical-optics part. This is confirmed by the plots in Fig. 8, where the average errors in the additive variant (22) and the Norton formula (27) are found as $0.22 \%$ and $0.16 \%$, respectively.

The oscillatory behavior over the initial part of the horizontal range observed in Fig. 8 is due to the interference of the direct and reflected waves in (23), with a lull in the oscillations near the pseudo-Brewster angle, where the reflected component has a sharp dip. This behavior may be 


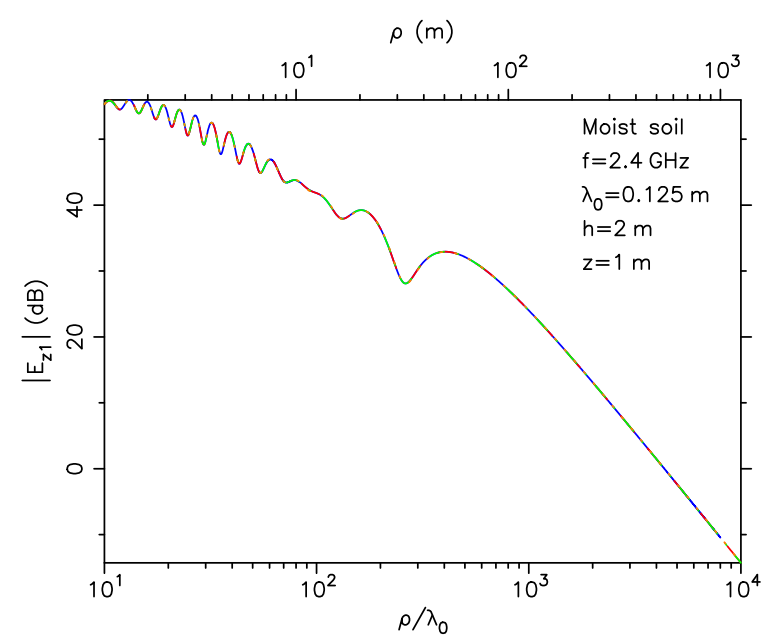

Fig. 8. As in Fig. 6, except for moist soil $\left(\varepsilon_{r}=15, \tan \delta=10^{-3}\right)$ at $2.4 \mathrm{GHz}$, with $h=2 \mathrm{~m}$ and $z=1 \mathrm{~m}$. The FEKO reference result is indicated by a full blue line, the additive saddle-point method asymptotic field (22) is plotted by a dashed red line, the positive-image multiplicative method (Norton formulation) field (27) is represented by a dot-dash green line, and the geometrical-optics field (23) is shown by a dotted orange line. These plots overlap and are indistinguishable in the figure. The surface wave terms are insignificant in this case and the total fields are essentially given by the geometrical-optics term.

explained by the approximate formula

$$
\left|\mathcal{E}_{G O}\right| \approx \frac{1}{k_{1} \rho} \sqrt{1+\left|\Gamma\left(\theta_{2}\right)\right|^{2}+2\left|\Gamma\left(\theta_{2}\right)\right| \cos \left(2 k_{1} z h / \rho-\psi\right)}
$$

applicable in the intermediate horizontal range, where we use the notation $\Gamma\left(\theta_{2}\right)=\left|\Gamma\left(\theta_{2}\right)\right| e^{j \psi}$. Note that the oscillations are superimposed on a $\rho^{-1}$ asymptote, which transitions to a $\rho^{-2}$ asymptote in the far zone, where the formula (35) applies. We should emphasize that these asymptotes are due to the interaction of the direct and reflected waves in $\mathcal{E}_{G O}$ and have nothing to do with the surface wave component, which is insignificant in this case.

In Fig. 9 we present error plots of the first-, second-, and third-order multiplicative negative-image methods for the case of Fig 8. We note that, unlike in all previous examples,

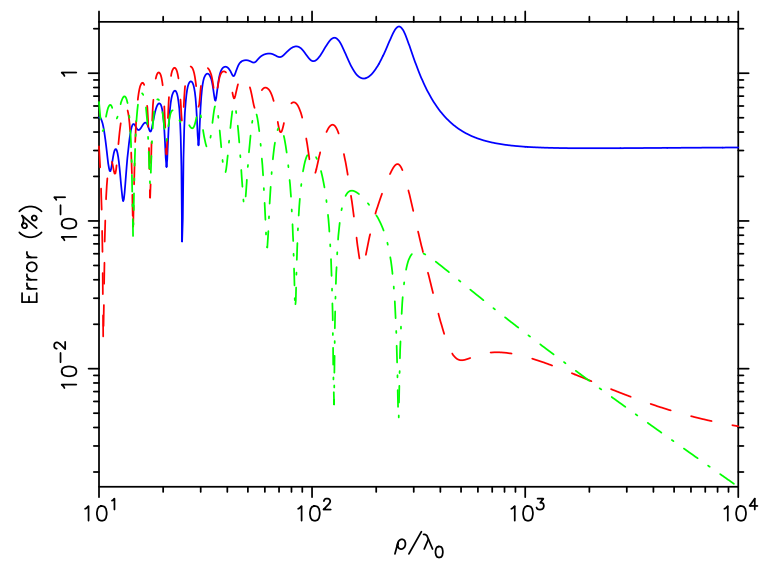

Fig. 9. As in Fig. 7, except for the elevated moist soil case of Fig. 8.

the first-order method does not break down completely, with the error remaining under approximately $2 \%$. However, this relatively good performance of the Ott-Clemmow formula is entirely attributable to the fact that the surface wave contribution is negligible in this case, with the field dominated by the geometrical-optics constituent.

We should mention in this context that the behavior of the surface wave component in the negative-image multiplicative Ott-Clemmow formula (28) has been used by Sarkar et al. [32]-[34] to explain an alleged $\rho^{-1.5}$ field asymptote in the intermediate range in cellular wireless communication. However, this reasoning is clearly untenable in view of the fact that the near-ground field behavior is dominated by the geometrical-optics component in most practical elevated transmitter-receiver configurations - as the last example clearly illustrates.

\section{Conclusion}

For a Hertzian dipole above or a lossy half-space medium, we have derived asymptotic field expressions by the first-order modified saddle-point method using either the additive pole treatment of van der Waerden, or the multiplicative variant of Ott and Clemmow. Furthermore, we have applied these procedures in conjunction with two equivalent formulations, which employ either positive or negative images of the dipole. We have found that, whereas the additive pole treatment leads to identical asymptotic field expressions irrespective of the sign of the image, the multiplicative variant yields distinct expressions for the two image configurations, which both differ from the unique result of the additive method. We have further found that the multiplicative method leads to the familiar Norton formula when used with the positive image, but with the negative image it results in a formula that predicts a zero surface field for a dipole located at the interface. We have confirmed these findings by numerical examples, which show that the additive method and the multiplicative method with the positive image yield excellent results of similar accuracy, but the first-order multiplicative negative-image variant is inaccurate and completely fails in the on-surface transmitterreceiver configuration.

\section{ApPendix A}

ANCILlaRY MATHEMATICAL DETAILS

We make use of the reference integrals [17, Sec. $4.2 \mathrm{~b}]$

$$
\int_{-\infty}^{\infty} e^{-\Omega_{2} s^{2}} d s=\sqrt{\frac{\pi}{\Omega_{2}}}
$$

and $[17$, Sec. $4.4 \mathrm{a}],[35$, Sec. 27.2]

$$
\begin{aligned}
s_{p} \int_{-\infty}^{\infty} \frac{e^{-\Omega_{2} s^{2}}}{s-s_{p}} d s & =2 s_{p}^{2} \int_{0}^{\infty} \frac{e^{-\Omega_{2} s^{2}}}{s^{2}-s_{p}^{2}} d s \\
& =-j \sqrt{\pi p} w(-\sqrt{p}) \sqrt{\frac{\pi}{\Omega_{2}}}
\end{aligned}
$$

where $w(\cdot)$ denotes the Faddeeva function [36] defined as [37, Eq. 7.2.3]

$$
w(z)=e^{-z^{2}} \operatorname{erfc}(-j z)=e^{-z^{2}} \frac{2}{\sqrt{\pi}} \int_{-j z}^{\infty} e^{-t^{2}} d t
$$


and where we have introduced the parameter

$$
p=\Omega_{2} s_{p}^{2}
$$

which corresponds to the "numerical distance" of Sommerfeld. We tacitly assume in (39) that $\mathfrak{I} m p<0$, which is always satisfied for non-plasmonic media [30].

The Sommerfeld-Norton attenuation function (20) has the ascending power-series representation [38]

$$
\mathcal{F}(p) \approx 1-j \sqrt{\pi p} e^{-p}-2 p+\frac{4 j}{3} p^{2}-\frac{8}{15} p^{3}+\cdots
$$

and the asymptotic expansion

$$
\mathcal{F}(p) \sim-2 j \sqrt{\pi p} e^{-p} \mathcal{U}(\mathfrak{J} m p)-\frac{1}{2 p}-\frac{3}{4 p^{2}}-\cdots
$$

where the first term only arises when $\mathfrak{I} m p>0$.

\section{ApPendix B}

\section{HIGHER-ORDER MULTIPLICATIVE METHODS}

The Ott-Clemmow method is based on the approximation (24), where only the leading term of the Maclaurin expansion of the integrand function is retained. To derive the secondand third-order multiplicative modified saddle-point methods, we retain up to three terms of the expansion, as follows [18]

$$
G_{\mp}(s)=\frac{1}{s-s_{p}}\left[\left(s-s_{p}\right) G_{\mp}(s)\right] \approx \frac{1}{s-s_{p}}\left(a_{0}+a_{1} s+a_{2} s^{2}\right)
$$

with the coefficients given as

$$
\begin{gathered}
a_{0}=-s_{p} g_{\mp}\left(\theta_{2}\right) \\
a_{1}=g_{\mp}\left(\theta_{2}\right)-\sqrt{2 j} s_{p} g_{\mp}^{\prime}\left(\theta_{2}\right) \\
a_{2}=\sqrt{2 j} g_{\mp}^{\prime}\left(\theta_{2}\right)-j \frac{s_{p}}{4}\left[g_{\mp}\left(\theta_{2}\right)+4 g_{\mp}^{\prime \prime}\left(\theta_{2}\right)\right]
\end{gathered}
$$

where the primes indicate differentiation. As before, the upper and lower signs pertain to the positive and negative image dipoles, respectively. Note that (44) reduces to (24) if only the first term of the expansion is retained. Upon using (44) with (45)-(47) in (12) and substituting into (2), for the second-order method we obtain

$$
\mathcal{E} \approx \mathcal{E}_{G O} \pm \sqrt{2 j} s_{p} g_{\mp}^{\prime}\left(\theta_{2}\right) \mathcal{F}(p) \frac{e^{-j \Omega_{2}}}{\Omega_{2}}
$$

and for the third-order method we obtain

$$
\mathcal{E} \approx \mathcal{E}_{G O} \pm j \frac{s_{p}^{2}}{4}\left\{\sin ^{2} \theta_{2}\left[1 \mp \Gamma\left(\theta_{2}\right)\right]+4 g_{\mp}^{\prime \prime}\left(\theta_{2}\right)\right\} \mathcal{F}(p) \frac{e^{-j \Omega_{2}}}{\Omega_{2}}
$$

where $\mathcal{E}_{G O}$ is the geometrical-optics field given by (23) and where

$$
\begin{array}{r}
g_{\mp}^{\prime}\left(\theta_{2}\right)=\sin ^{2} \theta_{2}\left\{\frac{5}{2} \cot \theta_{2}\left[1 \mp \Gamma\left(\theta_{2}\right)\right] \mp \Gamma^{\prime}\left(\theta_{2}\right)\right\} \\
g_{\mp}^{\prime \prime}(\xi)=\sin ^{2} \theta_{2}\left\{\frac{5}{4}\left(3 \cot ^{2} \theta_{2}-2\right)\left[1 \mp \Gamma\left(\theta_{2}\right)\right]\right. \\
\left.\mp\left[5 \cot \theta_{2} \Gamma^{\prime}\left(\theta_{2}\right)+\Gamma^{\prime \prime}\left(\theta_{2}\right)\right]\right\}
\end{array}
$$

with the derivatives of the reflection coefficient (7) given as

$$
\Gamma^{\prime}(\xi)=-\frac{2(\epsilon-1)}{\epsilon \kappa(\xi) \delta^{2}(\xi)} \sin \xi
$$

$$
\Gamma^{\prime \prime}(\xi)=\Gamma^{\prime}(\xi)\left\{\frac{\epsilon \cot \xi}{\kappa^{2}(\xi)}+2 \frac{\sin \xi}{\delta(\xi)}\left[1+\frac{\cos \xi}{\epsilon \kappa(\xi)}\right]\right\}
$$

where $\delta(\xi)=\cos \xi+\Delta(\xi)$ denotes the denominator of $\Gamma(\xi)$ and $\kappa(\xi)$ is defined in $(8)$.

It should be noted that our use of "order" refers to the number of terms retained in the expansion (44), and not to the powers of $\Omega_{2}^{-1}$ present in the asymptotic field formulas. In the latter sense of the word, the two- and three-term asymptotic field expansions presented here are still of the first order-as is the additive formula (22).

\section{ACKNOWLEDGMENT}

The authors acknowledge with gratitude the assistance of Minyu $\mathrm{Gu}$, who provided the Altair $\mathrm{FEKO}^{\mathrm{TM}}$ validation results.

\section{REFERENCES}

[1] A. Baños, Dipole Radiation in the Presence of a Conducting Half-Space. New York: Pergamon Press, 1966.

[2] J. R. Wait, "The ancient and modern history of EM ground-wave propagation," IEEE Antennas Propagat. Magaz., vol. 40, no. 5, pp. 7-24, 1998.

[3] R. E. Collin, "Hertzian dipole radiating over a lossy earth or sea: Some early and late 20th-century controversies," IEEE Antennas Propagat. Magaz., vol. 46, no. 2, pp. 64-79, 2004.

[4] A. Sommerfeld, "Über die Ausbreitung der Wellen in der drahtlosen Telegraphie," Ann. Physik, vol. 28, no. 4, pp. 665-736, 1909.

[5] — "Über die Ausbreitung der Wellen in der drahtlosen Telegraphie," Ann. Physik, vol. 81, pp. 1135-1153, 1926.

[6] K. A. Norton, "The propagation of radio waves over the surface of the earth and in the upper atmosphere-Part II: The propagation from vertical, horizontal, and loop antennas over a plane earth of finite conductivity," Proc. I.R.E., vol. 25, no. 9, pp. 1203-1237, 1937.

[7] B. van der Pol, "Theory of the reflection of the light from a point source by a finitely conducting flat mirror, with an application to radiotelegraphy," Physica, vol. 2, pp. 843-853, 1935.

[8] W. H. Wise, "Asymptotic dipole radiation formulas," Bell Syst. Techn. J., vol. 8, no. 4, pp. 662-671, 1929.

[9] H. Ott, "Die Sattelpunktsmethode in der Umgebung eines Pols Mit Anwendungen auf die Wellenoptik und Akustik," Ann. Physik, 5th Series, vol. 43, no. 6/7, pp. 393-403, 1943.

[10] W. Pauli, "On asymptotic series for functions in the theory of diffraction of light," Phys. Rev., vol. 54, no. 2, pp. 924-931, 1938.

[11] P. C. Clemmow, "Some extensions of the method of integration by steepest descents," Quart. J. Mech. Appl. Math., vol. 3, Pt. 2, pp. 241256, 1950.

[12] — The Plane Wave Spectrum Representation of Electromagnetic Fields. New York: Pergamon Press, 1966.

[13] J. R. Wait, "Theory of electromagnetic surface waves over geological conductors," Geofisica Pura e Applicata, vol. 28, no. 1, pp. 47-56, 1954.

[14] _ "Excitation of surface waves on conducting, stratified, dielectricclad, and corrugated surfaces," J. Res. Natl. Bur. Stand., vol. 59, no. 6, pp. 365-377, 1957.

[15] G. D. Bernard and A. Ishimaru, "On complex waves," Proc. IEE, vol. 114, no. 1 , pp. 43-49, 1967.

[16] B. L. van der Waerden, "On the method of saddle points," Appl. Sci. Res., vol. B2, pp. 33-45, 1951.

[17] L. B. Felsen and N. Marcuvitz, Radiation and Scattering of Waves. Englewood Cliffs, N.J.: Prentice Hall, 1973.

[18] K. A. Michalski and H. Lin, "On the Sommerfeld half-space problem: Appraisal of approximate solutions with extensions to plasmonics," $J$. Electromagn. Waves Appl., vol. 32, no. 4, pp. 483-503, 2018.

[19] — - "On the far-zone electromagnetic field of a vertical Hertzian dipole over an imperfectly conducting half-space with extensions to plasmonics," Radio Sci., vol. 52, pp. 798-810, 2017.

[20] K. A. Michalski and J. R. Mosig, "On the complete radiation pattern of a vertical Hertzian dipole above a low-loss ground (Invited Paper)," IEEE J. Microwaves, vol. 1, no. 3, pp. 747-762, 2021.

[21] K. A. Michalski, "On the efficient evaluation of integrals arising in the Sommerfeld halfspace problem," in Moment Methods in Antennas and Scatterers, R. C. Hansen, Ed. Boston: Artech House, 1990, pp. 325331, reprinted from IEE Proc., Pt. H: Microwaves Optics and Antennas, vol. 132, no. 5, pp. 213-218, 1985. 
[22] K. A. Norton, "The calculation of the ground-wave field intensity over a finitely conducting spherical earth," Proc. IRE, vol. 29, no. 12, pp. 623-639, 1941.

[23] T. S. M. Maclean and Z. Wu, Radiowave Propagation Over Ground. London: Chapman \& Hall, 1993.

[24] K. A. Michalski and D. R. Jackson, "Equivalence of the King and Norton-Bannister theories of dipole radiation over ground with extensions to plasmonics," IEEE Trans. Antennas Propagat., vol. 64, no. 12, pp. 5261-5261, 2016

[25] J. R. Wait, Electromagnetic Waves in Stratified Media, 2nd ed. New York: Pergamon Press, 1970.

[26] — Electromagnetic Wave Theory. New York: Harper \& Row, 1985.

[27] K. A. Michalski, "Extrapolation methods for Sommerfeld integral tails (Invited Paper)," IEEE Trans. Antennas Propagat., vol. 46, no. 10, pp. 1405-1418, 1998.

[28] K. A. Michalski and J. R. Mosig, "Efficient computation of Sommerfeld integral tails-methods and algorithms (Invited Paper)," J. Electromagn. Waves Appl., vol. 30, no. 3, pp. 281-317, 2016.

[29] A. Z. Elsherbeni, P. Nayeri, and C. J. Reddy, Antenna Analysis and Design Using FEKO Electromagnetic Simulation Software. Raleigh, NC: SciTech Publishing, 2014.

[30] K. A. Michalski and J. R. Mosig, "The Sommerfeld half-space problem revisited: From radio frequencies and Zenneck waves to visible light and Fano modes (Invited Paper)," J. Electromagn. Waves Appl., vol. 30, no. 1, pp. 1-42, 2016.

[31] M. H. Bezerra Cardoso, S. Mostarshedi, G. Boudoin, and J. Laheurte, "Analytical expressions for critical distances for near-ground propagation,” IEEE Trans. Antennas Propagat., vol. 66, no. 5, pp. 2482-2493, 2018.

[32] T. K. Sarkar, W. M. Dyab, M. N. Abdallah, M. Salazar-Palma, M. V. S. N. Prasad, S. W. Ting, and S. Barbin, "Electromagnetic macro modeling of propagation in mobile wireless communication: Theory and experiment," IEEE Antennas Propagat. Magaz., vol. 54, no. 6, pp. 17 43, 2012.

[33] T. K. Sarkar, M. Salazar-Palma, and M. N. Abdallah, The Physics and Mathematics of Electromagnetic Wave Propagation in Cellular Wireless Communication. Hoboken, NJ: Wiley, 2018.

[34] T. K. Sarkar and M. Salazar-Palma, "The demise of the hundred year old mythology of the famous Sommerfeld sign error along with the realization of the Zenneck wave and its relationship with surface, lateral and leaky waves," FERMAT, vol. 31, pp. 1-44, Article 1, Jul.-Aug. 2019.

[35] L. M. Brekhovskikh, Waves in Layered Media, 2nd ed. New York: Academic Press, 1980.

[36] G. P. M. Poppe and C. M. J. Wijers, "More efficient computation of the complex error function," ACM Trans. Math. Software, vol. 16, no. 1, pp. 38-46, 1990.

[37] F. W. J. Olver, D. W. Lozier, R. F. Boisvert, and C. W. Clark, NIST Handbook of Mathematical Functions. Cambridge, UK: Cambridge University Press, 2010.

[38] L. E. Vogler, "A note on the attenuation function for propagation over flat layered ground," IEEE Trans. Antennas Propagat., vol. 12, no. 2, pp. 240-242, 1964.

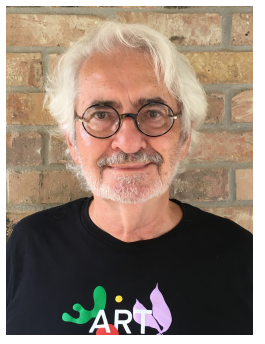

Krzysztof A. Michalski (S'78-M'81-SM'88-F'01) received the M.S. degree from the Wrocław Technological University, Poland, in 1974, and the Ph.D. degree from the University of Kentucky, Lexington, in 1981, both in electrical engineering. From 1982 to 1986 he was with the University of Mississippi, and since 1987 he has been with Texas A\&M University. He also held visiting professorships with École Polytechnique Fédérale de Lausanne (multiple times), Texas A\&M University at Qatar, Université de Nice-Sophia Antipolis, Universitat Politècnica de Catalunya, and Technische Universität München, and was a visiting scientist with Sandia National Laboratories, Albuquerque, NM, and the National Institute of Standards and Technology, Gaithersburg, MD. His research interests are in electromagnetic theory and computational electromagnetics, with emphasis on Green function methods and layered media problems. Dr. Michalski was the recipient the Best EMP Paper Award from the SUMMA Foundation (USA) in 1984, the Oliver Lodge Premium from the IEE (UK) in 1986, and the Sergei A. Schelkunoff Transactions Prize Paper Award from the IEEE Antennas and Propagation Society in 2015. He was Technical Program Chair of the 2002 IEEE Antennas and Propagation Society International Symposium.

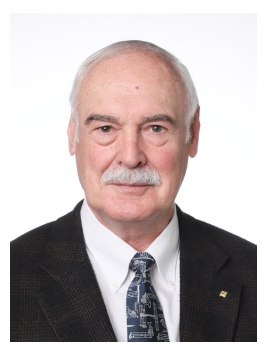

Juan R. Mosig (S'76-M'87-SM'94-F'99) was born in Cádiz, Spain. He received the Electrical Engineer degree from the Universidad Politécnica de Madrid, Madrid, Spain, in 1973, and the Ph.D. degree from the Ṕolytechnique Fédérale de Lausanne (EPFL), Lausanne, Switzerland, in 1983. In 1991, he was elected a Professor in the Laboratory of Electromagnetics and Acoustics (LEMA) at EPFL and its Director since 1999 until 2017, when he became Emeritus Professor. He has held scientific appointments with the RIT (Rochester, USA), the University of Colorado at Boulder (CO, USA), Universities of Rennes and Nice in France, DTU in Denmark and UTS in Australia. He has authored four chapters in books on planar antennas and circuits and more than 300 papers in peer-reviewed top international journals. He is the recipient of the 2015 IEEE APS Schelkunoff and the 2017 IEEE APWL Uslenghi Best Paper Awards. His research interests include electromagnetic theory, numerical methods, and planar antennas. Juan R. Mosig has been the Chair for the two COST (European Cooperation in Science \& Technology) Actions and a member of the COST Scientific Committee (2003-2011). He is a founding member of the European Association on Antennas and Propagation (EurAAP) and he was Chair of the 2006 and 2016 editions of the EuCAP Conferences. Among his awards and distinctions, he is the recipient of the JR James Lifetime Career Award (LAPC, UK, 2015), the EurAAP Award (2016) and the Japanese ISAP Appreciation Award (2016). He is also a Member of the Swiss Academy of Technical Sciences (SATW) and Life Fellow of IEEE. 